\title{
A Unified Approach to Some Classes of Nonlinear Integral Equations
}

\author{
Nurgali K. Ashirbayev, ${ }^{1}$ Józef Banas, ${ }^{2}$ and Raina Bekmoldayeva ${ }^{1}$ \\ ${ }^{1}$ Department of Mathematics, Science-Pedagogical Faculty, M. Auezov South Kazakhstan State University, Tauke Khan Avenue 5, \\ Shymkent 160012, Kazakhstan \\ ${ }^{2}$ Department of Mathematics, Rzeszów University of Technology, Al. Powstańców Warszawy 8, 35-959 Rzeszów, Poland
}

Correspondence should be addressed to Józef Banaś; jbanas@prz.edu.pl

Received 30 July 2014; Accepted 20 August 2014; Published 3 September 2014

Academic Editor: Kishin Sadarangani

\begin{abstract}
Copyright ( $\odot 2014$ Nurgali K. Ashirbayev et al. This is an open access article distributed under the Creative Commons Attribution License, which permits unrestricted use, distribution, and reproduction in any medium, provided the original work is properly cited.

We are going to discuss some important classes of nonlinear integral equations such as integral equations of Volterra-Chandrasekhar type, quadratic integral equations of fractional orders, nonlinear integral equations of Volterra-Wiener-Hopf type, and nonlinear integral equations of Erdélyi-Kober type. Those integral equations play very significant role in applications to the description of numerous real world events. Our aim is to show that the mentioned integral equations can be treated from the view point of nonlinear Volterra-Stieltjes integral equations. The Riemann-Stieltjes integral appearing in those integral equations is generated by a function of two variables. The choice of a suitable generating function enables us to obtain various kinds of integral equations. Some results concerning nonlinear Volterra-Stieltjes integral equations in several variables will be also discussed.
\end{abstract}

\section{Introduction}

In the theory of integral equations and their numerous applications, one can encounter some classes of integral equations having an important significance. This fact is mainly connected with applications of the mentioned classes of integral equations to the description of several real world events which appear in engineering, mechanics, physics, mathematical physics, electrochemistry, bioengineering, porous media, viscoelasticity, control theory, transport theory, kinetic theory of gases, radiative transfer, and other important branches of exact science and applied mathematics (cf. [1-12]).

Let us distinguish and describe some important classes of nonlinear integral equations mentioned tacitly above.

The first class we are going to present is the class of the so-called quadratic integral equations of VolterraChandrasekhar type (see $[1,7,13,14]$, e.g.). The interest in the study of those integral equations was initiated around 1950 by the famous astrophysicist Chandrasekhar [1], who investigated the following integral equation:

$$
x(t)=l+x(t) \int_{0}^{1} \frac{t}{t+s} \varphi(s) x(s) d s,
$$

being the so-called quadratic (nonlinear) integral equation and called the Chandrasekhar integral equation.

Nowadays, integral equation (1) has been generalized in a few directions but in general two principal types of generalizations of (1) are investigated, namely, the quadratic integral equation of Fredholm-Chandrasekhar type

$$
x(t)=a(t)+f(t, x(s)) \int_{0}^{a} \frac{v(t, s, x(s))}{t+s} d s
$$

and the quadratic integral equation of Volterra-Chandrasekhar type

$$
x(t)=a(t)+f(t, x(t)) \int_{0}^{t} \frac{v(t, s, x(s))}{t+s} d s .
$$

We will focus on integral equations having form (3), that is, on nonlinear integral equations of Volterra-Chandrasekhar type.

The second class of nonlinear integral equations which will be discussed is the class of the so-called nonlinear integral equations of fractional order. Such equations have the form

$$
x(t)=a(t)+\frac{f(t, x(t))}{\Gamma(\alpha)} \int_{a}^{t} \frac{v(t, s, x(s))}{(t-s)^{1-\alpha}} d s,
$$


where $\alpha \in(0,1)$ is a fixed number and $\Gamma(\alpha)$ denotes the gamma function.

Observe that (4) is the so-called singular integral equation (of Abel type). These equations were very intensively studied during the last three decades and found a vast number of applications. Mathematicians working in the theory of integral equations of fractional orders wrote several papers and monographs devoted to those equations $[4,5,8-11,15-$ $20]$.

The next, third class of nonlinear integral equations which we would like to present, is associated with the so-called nonlinear integral equations of Volterra-Wiener-Hopf type. Such equations are a special case of integral equations with kernels depending on the difference of arguments and they also play very important role in applications (cf. [3, 12, 2123]).

The Volterra-Wiener-Hopf integral equation has the form

$$
x(t)=a(t)+\int_{0}^{t} k(t-s) v(s, x(s)) d s
$$

where $t \in \mathbb{R}_{+}=[0, \infty)$ or $t \in[0, T]$ with $T>0$.

Now, let us describe the fourth class of nonlinear integral equations being the object of our study as well as being recently very intensively investigated with regard to its numerous applications [24-30]. That class comprises integral equations called the nonlinear Erdélyi-Kober integral equations and having the form

$$
x(t)=a(t)+\frac{1}{\Gamma(\alpha)} \int_{0}^{t} \frac{m s^{m-1} s^{p} v(t, s, x(s))}{\left(t^{m}-s^{m}\right)^{1-\alpha}} d s,
$$

where $\alpha, m$, and $p$ are positive constant and $\alpha \in(0,1)$. Moreover, $t \in I=[0,1]$ (or $I=[a, b])$.

Obviously, the integral equation of Erdélyi-Kober type creates the generalization of the integral equation of fractional order (4). Indeed, putting in (6) $m=1$ and including the factor $s^{p}$ into the function $v(t, s, x)$, we obtain (4) with $f(t, x) \equiv 1$.

Our aim in this paper is to show that all four classes of nonlinear integral equations (3)-(6) can be treated from one point of view. More precisely, we show that with help of nonlinear Volterra-Stieltjes integral equations we are able to unify all those classes in such a way that they are particular cases of the mentioned Volterra-Stieltjes integral equations.

The paper has a review character and is based on the results from $[14,21,25,31]$.

\section{Notation, Definitions, and Auxiliary Results}

In this section, we provide notation, definitions, and auxiliary results which will be needed in our further considerations. Firstly, we recall a few facts concerning functions of bounded variation [32]. Thus, assume that $x$ is a real function defined on the fixed interval $[a, b]$. Then, the symbol $\bigvee_{a}^{b} x$ denotes the variation of the function $x$ on the interval $[a, b]$. If $\bigvee_{a}^{b} x<\infty$, we say that $x$ is of bounded variation on $[a, b]$. Similarly, if we have a function $u(t, s)=u:[a, b] \times[c, d] \rightarrow \mathbb{R}$, then we denote by $\bigvee_{t=p}^{q} u(t, s)$ the variation of the function $t \rightarrow u(t, s)$ on the interval $[p, q] \subset[a, b]$, where $s$ is a fixed number in $[c, d]$. In a similar way, we define the quantity $\bigvee_{s=p}^{q} u(t, s)$.

Now, assume that $x$ and $\varphi$ are two real functions defined on the interval $[a, b]$. Then, we can define the Stieltjes integral (in the Riemann-Stieltjes sense)

$$
\int_{a}^{b} x(t) d \varphi(t)
$$

under appropriate assumptions on the functions $x$ and $\varphi$ (cf. [32]). For example, if we require that $x$ is continuous and $\varphi$ is of bounded variation on $[a, b]$, then the Stieltjes integral (7) does exist [32].

Let us mention that in our considerations we will often use the following two important lemmas [32].

Lemma 1. If $x$ is Stieltjes integrable on the interval $[a, b]$ with respect to a function $\varphi$ of bounded variation, then

$$
\left|\int_{a}^{b} x(t) d \varphi(t)\right| \leq \int_{a}^{b}|x(t)| d\left(\bigvee_{a}^{t} \varphi\right) .
$$

Lemma 2. Let $x_{1}, x_{2}$ be Stieltjes integrable functions on the interval $[a, b]$ with respect to a nondecreasing function $\varphi$, such that $x_{1}(t) \leq x_{2}(t)$ for $t \in[a, b]$. Then,

$$
\int_{a}^{b} x_{1}(t) d \varphi(t) \leq \int_{a}^{b} x_{2}(t) d \varphi(t) .
$$

Obviously, in a similar way we can also consider Stieltjes integrals of the form

$$
\int_{a}^{b} x(s) d_{s} g(t, s),
$$

where $g:[a, b] \times[a, b] \rightarrow \mathbb{R}$ and the symbol $d_{s}$ indicates the integration with respect to $s$. The details concerning the integral of this type will be given later.

Now, assume that $x$ is a real function defined on the interval $[a, b]$. Denote by $\omega(x, \varepsilon)$ the modulus of continuity of the function $x$ defined by the formula

$$
\begin{aligned}
& \omega(x, \varepsilon)=\sup \{|x(t)-x(s)|: \\
& \quad t, s \in[a, b],|t-s| \leq \varepsilon\} .
\end{aligned}
$$

Similarly, if $p(t, s)=p:[a, b] \times[c, d] \rightarrow \mathbb{R}$, then we can define the modulus of continuity of the function $p(t, s)$ with respect to each variable separately. For example,

$$
\begin{aligned}
& \omega(p(t, \cdot), \varepsilon)=\sup \{|p(t, u)-p(t, v)|: \\
&u, v \in[c, d],|u-v| \leq \varepsilon\},
\end{aligned}
$$

where $t$ is a fixed number in the interval $[a, b]$.

In what follows, we recall some facts concerning measures of noncompactness which will be used later on [33].

To this end, assume that $E$ is an infinite dimensional Banach space with the norm $\|\cdot\|$ and zero element $\theta$. Denote by $B(x, r)$ the closed ball centered at $x$ and of radius $r$. The symbol $B_{r}$ will denote the ball $B(\theta, r)$. 
For a given nonempty bounded subset $X$ of $E$, we denote by $\chi(X)$ the so-called Hausdorff measure of noncompactness of the set $X$ [33]. This quantity is defined by the formula

$$
\chi(X)=\inf \{\varepsilon>0: X \text { has a finite } \varepsilon \text {-net in } E\} .
$$

Let us mention that the function $\chi$ has several useful properties and is often applied in nonlinear analysis [33]. Obviously, the concept of a measure of noncompactness may be defined in a more general way $[33,34]$, but for our purposes the Hausdorff measure of noncompactness defined by (13) will be completely sufficient.

Indeed, in our further considerations, we will work in the Banach space $C(I)$ consisting of real functions defined and continuous on the interval $I=[a, b]$, with the standard maximum norm. If $X$ is a nonempty and bounded subset of $C(I)$, then the Hausdorff measure of noncompactness of $X$ can be expressed by the formula [33]

$$
\chi(X)=\frac{1}{2} \omega_{0}(X)
$$

where

$$
\omega_{0}(X)=\lim _{\varepsilon \rightarrow 0} \omega(X, \varepsilon)
$$

and the symbol $\omega(X, \varepsilon)$ stands for the modulus of continuity of the set $X$ defined in the following way:

$$
\omega(X, \varepsilon)=\sup \{\omega(x, \varepsilon): x \in X\} .
$$

In our further considerations, we will utilize the fixed point theorem of Darbo type [33], which is formulated below.

Theorem 3. Let $\Omega$ be a nonempty, bounded, closed, and convex subset of the space $E$ and let $Q: \Omega \rightarrow \Omega$ be a continuous mapping such that there exists a constant $k \in[0,1)$ for which $\chi(Q X) \leq k \chi(X)$ for an arbitrary nonempty subset $X$ of $\Omega$. Then, $Q$ has at least one fixed point in the set $\Omega$.

Further on, we recall some facts concerning the so-called superposition operator [35]. To this end, assume that $I=$ $[a, b]$ and $f:[a, b] \times \mathbb{R} \rightarrow \mathbb{R}$ is a given function. Then, to every function $x: I \rightarrow \mathbb{R}$, we may assign the function $F x$ defined by the formula

$$
(F x)(t)=f(t, x(t))
$$

for $t \in I$. The operator $F$ defined in such a way is called the superposition operator generated by the function $f=f(t, x)$. For our further purposes, we will need the following result concerning the behaviour of the superposition operator $F$ in the space $C(I)[35]$.

Lemma 4. The superposition operator $F$ defined by (17) transforms the space $C(I)$ into itself and is continuous if and only if the function $f$ generating the operator $F$ is continuous on the set $I \times \mathbb{R}$.

\section{A Nonlinear Volterra-Stieltjes Integral Equation and Its Special Cases}

The considerations of this section are focused on the following nonlinear Volterra-Stieltjes integral equation:

$$
x(t)=a(t)+\frac{f(t, x(t))}{\Gamma(\alpha)} \int_{0}^{t} v(t, s, x(s)) d_{s} g(t, s),
$$

where $t \in I=[0,1]$ and $\Gamma(\alpha)$ (similarly as earlier) denotes the gamma function. Moreover, $\alpha$ is a fixed number in the interval $(0,1)$. Let us notice that the interval $[0,1]$ can be replaced by any interval $[a, b]$.

The details concerning assumptions imposed on the components of (18) will be given later. Now, we show that integral equation (18) unifies all previously considered integral equations (3)-(6).

At the beginning, denote by $\Delta$ the triangle

$$
\Delta=\{(t, s): 0 \leq s \leq t \leq 1\},
$$

and consider the function $g(t, s)=g: \Delta \rightarrow \mathbb{R}$ defined in the following way:

$$
g(t, s)= \begin{cases}t \ln \frac{t+s}{t} & \text { for } 0<s \leq t \\ 0 & \text { for } t=0\end{cases}
$$

It is easy to see that the above function $g(t, s)$ is continuous on the triangle $\Delta$. On the other hand, we get

$$
d_{s} g(t, s)=\left(\frac{\partial}{\partial s} g(t, s)\right) d s=\frac{t}{t+s} d s
$$

Hence, we see that the integral equation of VolterraChandrasekhar type (3) (or (1), in the simplest case) can be treated as a special case of (18).

Further, consider the function $g(t, s)$ defined by the formula

$$
g(t, s)=\frac{1}{\alpha}\left[t^{\alpha}-(t-s)^{\alpha}\right],
$$

where $(t, s) \in \Delta$. Obviously, we have

$$
d_{s} g(t, s)=\frac{1}{(t-s)^{1-\alpha}} d s,
$$

which shows that the integral equation of fractional order (4) is also a particular case of (18).

To show that the Volterra-Wiener-Hopf integral equation (5) is a special case of (18), let us consider the function $g(t, s)$ given by the formula

$$
g(t, s)=\int_{0}^{s} k(t-z) d z,
$$

under appropriate assumptions imposed on the function $k=$ $k(u)$ (cf. [21]). Obviously, we have

$$
d_{s} g(t, s)=\frac{\partial}{\partial s}\left(\int_{0}^{s} k(t-z) d z\right) d s=k(t-s) d s,
$$

and we see that (5) is in fact a special case of (18). 
Finally, let us take into account the nonlinear ErdélyiKober integral equation (6). Then, putting

$$
g(t, s)=t^{\alpha m}-\left(t^{m}-s^{m}\right)^{\alpha}
$$

for $(t, s) \in \Delta$, we have that

$$
d_{s} g(t, s)=\frac{\alpha m s^{m-1}}{\left(t^{m}-s^{m}\right)^{1-\alpha}} d s .
$$

Thus, we see that the integral equation (6) is also a special case of (18).

Now, we formulate theorem on the existence of solutions of Volterra-Stieltjes integral equation (18) imposing assumptions of such a type that the obtained theorem will ensure also the existence of solutions of all particular cases of (18) mentioned above.

We will consider the existence of solutions of (18) under the following hypotheses.

(i) The function $a=a(t)$ is continuous on the interval $I$.

(ii) The function $f(t, x)=f: I \times \mathbb{R} \rightarrow \mathbb{R}$ is continuous and satisfies the Lipschitz condition with respect to the second variable; that is, there exists a constant $k>$ 0 such that

$$
|f(t, x)-f(t, y)| \leq k|x-y|
$$

for all $t \in I$ and $x, y \in \mathbb{R}$.

(iii) The function $g(t, s)=g: \Delta \rightarrow \mathbb{R}$ is continuous.

(iv) The function $s \rightarrow g(t, s)$ is of bounded variation on the interval $[0, t]$ for each fixed $t \in I$.

(v) For any $\varepsilon>0$, there exists $\delta>0$ such that, for all $t_{1}, t_{2} \in I, t_{1}<t_{2}$, and $t_{2}-t_{1} \leq \delta$, the following inequality holds:

$$
\bigvee_{s=0}^{t_{1}}\left[g\left(t_{2}, s\right)-g\left(t_{1}, s\right)\right] \leq \varepsilon .
$$

(vi) $g(t, 0)=0$ for any $t \in I$.

(vii) $v: \Delta \times \mathbb{R} \rightarrow \mathbb{R}$ is continuous such that $|v(t, s, x)| \leq$ $\phi(|x|)$ for all $(t, s) \in \Delta$ and for each $x \in \mathbb{R}$, where $\phi: \mathbb{R}_{+} \rightarrow \mathbb{R}_{+}$is a nondecreasing function.

Now, we provide a few properties of the function $g=$ $g(t, s)$ which will be needed in our further considerations. Obviously, we will assume that $g$ satisfies assumptions (iii)(vi).

Let us notice that these properties were proved in [14].

Lemma 5. Let assumptions (iii)-(v) be satisfied. Then, for an arbitrarily fixed number $t_{2} \in I\left(t_{2}>0\right)$ and for any $\varepsilon>0$, there exists $\delta>0$ such that if $t_{1}, t_{2} \in I, t_{1}<t_{2}$, and $t_{2}-t_{1} \leq \delta$ then

$$
\bigvee_{s=t_{1}}^{t_{2}} g\left(t_{2}, s\right) \leq \varepsilon .
$$

Lemma 6. Under assumptions (iii)-(v), the function

$$
t \longrightarrow \bigvee_{s=0}^{t} g(t, s)
$$

is continuous on the interval I.

Corollary 7. There exists a finite positive constant $K$ such that

$$
K=\sup \left\{\bigvee_{s=0}^{t} g(t, s): t \in I\right\} \text {. }
$$

In fact, the above statement is an immediate consequence of the continuity of the function

$$
t \longrightarrow \bigvee_{s=0}^{t} g(t, s) .
$$

Further, let us denote by $F_{1}$ the finite constant (cf. assumption (iii)) defined by the formula

$$
F_{1}=\max \{|f(t, 0)|: t \in I\} .
$$

Now, we are prepared to formulate the last assumption utilized in our considerations.

(viii) There exists a positive solution $r_{0}$ of the inequality

$$
\|a\|+K\left(k r+F_{1}\right) \phi(r) \leq r,
$$

such that $k K \phi\left(r_{0}\right)<1$.

Our main result is formulated in the form of the following theorem.

Theorem 8. Under assumptions (i)-(viii), there exists at least one solution $x=x(t)$ of (18) belonging to the space $C(I)$.

Proof. At the beginning, let us introduce two functions $M(\varepsilon)$, $N(\varepsilon)$ defined in the following way:

$$
\begin{gathered}
M(\varepsilon)=\sup \left\{\bigvee_{s=0}^{t_{1}}\left[g\left(t_{2}, s\right)-g\left(t_{1}, s\right)\right]:\right. \\
\left.t_{1}, t_{2} \in I, t_{1}<t_{2}, t_{2}-t_{1} \leq \varepsilon\right\}, \\
N(\varepsilon)=\sup \left\{\bigvee_{s=t_{1}}^{t_{2}} g\left(t_{2}, s\right): t_{1}, t_{2} \in I, t_{1}<t_{2}, t_{2}-t_{1} \leq \varepsilon\right\} .
\end{gathered}
$$

Notice that in view of assumption (v) we have that $M(\varepsilon) \rightarrow 0$ as $\varepsilon \rightarrow 0$. Similarly, $N(\varepsilon) \rightarrow 0$ as $\varepsilon \rightarrow 0$ which is an easy consequence of Lemma 5 .

Next, for a fixed $x \in C(I)$ and $t \in I$, let us denote

$$
\begin{aligned}
& (F x)(t)=f(t, x(t)), \\
& (V x)(t)=\int_{0}^{t} v(t, s, x(s)) d_{s} g(t, s), \\
& (Q x)(t)=a(t)+(F x)(t)(V x)(t) .
\end{aligned}
$$


Further, fix arbitrarily $\varepsilon>0$ and take $t_{1}, t_{2} \in I$ such that $t_{1}<t_{2}$ and $t_{2}-t_{1} \leq \varepsilon$. Then, in view of our assumptions and Lemmas 1 and 2, for a fixed $x \in C(I)$, we obtain

$$
\begin{aligned}
& \left|(V x)\left(t_{2}\right)-(V x)\left(t_{1}\right)\right| \\
& \leq\left|\int_{0}^{t_{2}} v\left(t_{2}, s, x(s)\right) d_{s} g\left(t_{2}, s\right)-\int_{0}^{t_{1}} v\left(t_{2}, s, x(s)\right) d_{s} g\left(t_{2}, s\right)\right| \\
& +\mid \int_{0}^{t_{1}} v\left(t_{2}, s, x(s)\right) d_{s} g\left(t_{2}, s\right) \\
& -\int_{0}^{t_{1}} v\left(t_{1}, s, x(s)\right) d_{s} g\left(t_{2}, s\right) \\
& +\mid \int_{0}^{t_{1}} v\left(t_{1}, s, x(s)\right) d_{s} g\left(t_{2}, s\right) \\
& -\int_{0}^{t_{1}} v\left(t_{1}, s, x(s)\right) d_{s} g\left(t_{1}, s\right) \\
& \leq \int_{t_{1}}^{t_{2}}\left|v\left(t_{2}, s, x(s)\right)\right| d_{s}\left(\bigvee_{p=0}^{s} g\left(t_{2}, p\right)\right) \\
& +\int_{0}^{t_{1}}\left|v\left(t_{2}, s, x(s)\right)-v\left(t_{1}, s, x(s)\right)\right| d_{s}\left(\bigvee_{p=0}^{s} g\left(t_{2}, p\right)\right) \\
& +\int_{0}^{t_{1}}\left|v\left(t_{1}, s, x(s)\right)\right| d_{s}\left(\bigvee_{p=0}^{s}\left[g\left(t_{2}, p\right)-g\left(t_{1}, p\right)\right]\right) \\
& \leq \phi(\|x\|) \int_{t_{1}}^{t_{2}} d_{s}\left(\bigvee_{p=0}^{s} g\left(t_{2}, p\right)\right) \\
& +\int_{0}^{t_{1}}\left|v\left(t_{2}, s, x(s)\right)-v\left(t_{1}, s, x(s)\right)\right| d_{s}\left(\bigvee_{p=0}^{s} g\left(t_{2}, p\right)\right) \\
& +\phi(\|x\|) \int_{0}^{t_{1}} d_{s}\left(\bigvee_{p=0}^{s}\left[g\left(t_{2}, p\right)-g\left(t_{1}, p\right)\right]\right) \\
& \leq \phi(\|x\|)\left[\bigvee_{s=0}^{t_{2}} g\left(t_{2}, s\right)-\bigvee_{s=0}^{t_{1}} g\left(t_{2}, s\right)\right] \\
& +\omega(\varepsilon) \bigvee_{s=0}^{t_{1}} g\left(t_{2}, s\right)+\phi(\|x\|) \bigvee_{s=0}^{t_{1}}\left[g\left(t_{2}, s\right)-g\left(t_{1}, s\right)\right] \\
& \leq \phi(\|x\|) \bigvee_{s=t_{1}}^{t_{2}} g\left(t_{2}, s\right) \\
& +\omega(\varepsilon) \bigvee_{s=0}^{t_{2}} g\left(t_{2}, s\right)+\phi(\|x\|) M(\varepsilon) \\
& \leq \phi(\|x\|) N(\varepsilon)+K \omega(\varepsilon)+\phi(\|x\|) M(\varepsilon),
\end{aligned}
$$

where we denoted

$$
\begin{aligned}
& \omega(\varepsilon) \\
& =\sup \left\{\left|v\left(t_{2}, s, y\right)-v\left(t_{1}, s, y\right)\right|:\right. \\
& \left.\qquad\left(t_{1}, s\right),\left(t_{2}, s\right) \in \Delta,\left|t_{2}-t_{1}\right| \leq \varepsilon, y \in[-\|x\|,\|x\|]\right\} .
\end{aligned}
$$

Moreover, the functions $M(\varepsilon), N(\varepsilon)$ are defined by (36) and the constant $K$ is defined by (32).

Observe that in view of the uniform continuity of the function $v$ on the set $\Delta \times[-\|x\|,\|x\|]$ we infer that $\omega(\varepsilon) \rightarrow 0$ as $\varepsilon \rightarrow 0$. Linking this fact with Lemma 5 and the properties of the functions $M(\varepsilon)$ and $N(\varepsilon)$ indicated previously, we deduce from (38) that the function $V x$ is continuous on the interval I.

On the other hand, the function $F x$ is continuous on $I$ which is an easy consequence of assumption (ii) and Lemma 4. Thus, keeping in mind the above established facts, assumption (i), and (37), we conclude that the function $Q x$ is continuous on the interval $I$. This means that the operator $Q$ transforms the space $C(I)$ into itself.

In what follows, we show that the operator $Q$ is continuous on the space $C(I)$. To this end, let us first observe that in view of the properties of the superposition operator $F$ (cf. Lemma 4) it is sufficient to show that the operator $V$ defined by (37) is continuous on $C(I)$.

To do this, fix $\varepsilon>0$ and $x \in C(I)$. Next, take an arbitrary function $y \in C(I)$ with $\|x-y\| \leq \varepsilon$. Then, in view of Lemma 1 , for an arbitrary fixed $t \in I$, we obtain

$$
\begin{aligned}
& |(V x)(t)-(V y)(t)| \\
& \quad \leq \int_{0}^{t}|v(t, s, x(s))-v(t, s, y(s))| d_{s}\left(\bigvee_{p=0}^{s} g(t, p)\right) .
\end{aligned}
$$

Now, let us denote

$$
\begin{gathered}
P=\|x\|+\varepsilon, \\
\omega_{P}(v, \varepsilon)=\sup \{|v(t, s, w)-v(t, s, u)|: \\
(t, s) \in \Delta, w, u \in[-P, P],|w-u| \leq \varepsilon\} .
\end{gathered}
$$

Then, from (40), we derive the following inequalities:

$$
\begin{aligned}
& |(V x)(t)-(V y)(t)| \\
& \quad \leq \int_{0}^{t} \omega_{P}(v, \varepsilon) d_{s}\left(\bigvee_{z=0}^{s} g(t, z)\right) \\
& \quad \leq \omega_{P}(v, \varepsilon) \bigvee_{s=0}^{t} g(t, s) \leq K \omega_{P}(v, \varepsilon) .
\end{aligned}
$$

Hence, in virtue of the uniform continuity of the function $v$ on the set $\Delta \times[-P, P]$, we deduce that $V$ is continuous on the space $C(I)$. 
In what follows, let us fix arbitrarily $x \in C(I)$. Then, taking into account the imposed assumptions and applying Lemmas 1 and 2 , for a fixed $t \in I$, we get

$|(Q x)(t)| \leq|a(t)|$

$$
\begin{aligned}
& \quad+|f(t, x(t))| \int_{0}^{t}|v(t, s, x(s))| d_{s}\left(\bigvee_{p=0}^{s} g(t, p)\right) \\
& \leq\|a\|+[|f(t, x(t))-f(t, 0)|+|f(t, 0)|] \\
& \quad \times \int_{0}^{t} \phi(\|x\|) d_{s}\left(\bigvee_{p=0}^{s} g(t, p)\right) \\
& \leq\|a\|+\left(k\|x\|+F_{1}\right) \phi(\|x\|) \bigvee_{s=0}^{t} g(t, s) .
\end{aligned}
$$

Hence, in view of Corollary 7, we derive the following estimate:

$$
\|Q x\| \leq\|a\|+\left(k\|x\|+F_{1}\right) K \phi(\|x\|) .
$$

Then, keeping in mind assumption (viii), we deduce that there exists a number $r_{0}$ such that $Q$ transforms the ball $B_{r_{0}}$ into itself and $k K \phi\left(r_{0}\right)<1$.

In what follows, let us take a nonempty subset $X$ of the ball $B_{r_{0}}$ and $x \in X$. Next, fix $\varepsilon>0$ and choose $t_{1}, t_{2} \in I$ such that $t_{1}<t_{2}$ and $t_{2}-t_{1} \leq \varepsilon$. Then, applying (38), we obtain

$$
\begin{aligned}
\mid(Q x) & \left(t_{2}\right)-(Q x)\left(t_{1}\right) \mid \\
\leq & \left|a\left(t_{2}\right)-a\left(t_{1}\right)\right| \\
+ & \left|(F x)\left(t_{2}\right)(V x)\left(t_{2}\right)-(F x)\left(t_{2}\right)(V x)\left(t_{1}\right)\right| \\
+ & \left|(F x)\left(t_{2}\right)(V x)\left(t_{1}\right)-(F x)\left(t_{1}\right)(V x)\left(t_{1}\right)\right| \\
\leq & \omega(a, \varepsilon) \\
+ & \left|(F x)\left(t_{2}\right)\right|\left|(V x)\left(t_{2}\right)-(V x)\left(t_{1}\right)\right| \\
+ & \left|(V x)\left(t_{1}\right)\right|\left|(F x)\left(t_{2}\right)-(F x)\left(t_{1}\right)\right| \\
\leq & \omega(a, \varepsilon)+\left[\left|f\left(t_{2}, x\left(t_{2}\right)\right)-f\left(t_{2}, 0\right)\right|+\left|f\left(t_{2}, 0\right)\right|\right] \\
\times & \{\phi(\|x\|) N(\varepsilon)+K \omega(\varepsilon)+\phi(\|x\|) M(\varepsilon)\} \\
+ & \left|\int_{0}^{t_{1}} v\left(t_{1}, s, x(s)\right) d_{s} g\left(t_{1}, s\right)\right| \\
\times & \left\{\left|f\left(t_{2}, x\left(t_{2}\right)\right)-f\left(t_{2}, x\left(t_{1}\right)\right)\right|\right. \\
& \left.+\left|f\left(t_{2}, x\left(t_{1}\right)\right)-f\left(t_{1}, x\left(t_{1}\right)\right)\right|\right\}
\end{aligned}
$$

$$
\begin{aligned}
\leq & \omega(a, \varepsilon)+\left(k\|x\|+F_{1}\right) \\
& \times\{\phi(\|x\|) N(\varepsilon)+K \omega(\varepsilon)+\phi(\|x\|) M(\varepsilon)\} \\
& +\int_{0}^{t_{1}}\left|v\left(t_{1}, s, x(s)\right)\right| d_{s}\left(\bigvee_{p=0}^{s} g\left(t_{1}, p\right)\right) \\
& \times\left\{k\left|x\left(t_{2}\right)-x\left(t_{1}\right)\right|+\omega_{r_{0}}^{1}(f, \varepsilon)\right\},
\end{aligned}
$$

where we denoted

$$
\begin{aligned}
& \omega_{r_{0}}^{1}(f, \varepsilon)=\sup \left\{\left|f\left(t_{2}, x\right)-f\left(t_{1}, x\right)\right|:\right. \\
&\left.t_{1}, t_{2} \in I,\left|t_{2}-t_{1}\right| \leq \varepsilon, x \in\left[-r_{0}, r_{0}\right]\right\} .
\end{aligned}
$$

Further, from (45), we get

$$
\begin{aligned}
\mid(Q x) & \left(t_{2}\right)-(Q x)\left(t_{1}\right) \mid \\
\leq & \omega(a, \varepsilon)+\left(k r_{0}+F_{1}\right) \\
& \times\left\{\phi\left(r_{0}\right) N(\varepsilon)+K \omega(\varepsilon)+\phi\left(r_{0}\right) M(\varepsilon)\right\} \\
& +\phi\left(r_{0}\right) \int_{0}^{t_{1}} d_{s}\left(\bigvee_{p=0}^{s} g\left(t_{1}, p\right)\right)\left\{k \omega(x, \varepsilon)+\omega_{r_{0}}^{1}(f, \varepsilon)\right\} \\
\leq & \omega(a, \varepsilon)+\left(k r_{0}+F_{1}\right) \\
& \times\left\{\phi\left(r_{0}\right) N(\varepsilon)+K \omega(\varepsilon)+\phi\left(r_{0}\right) M(\varepsilon)\right\} \\
& +K \phi\left(r_{0}\right)\left\{k \omega(x, \varepsilon)+\omega_{r_{0}}^{1}(f, \varepsilon)\right\} .
\end{aligned}
$$

Hence, we have

$$
\begin{aligned}
& \omega(Q x, \varepsilon) \\
& \leq \omega(a, \varepsilon)+\left(k r_{0}+F_{1}\right) \\
& \times\left\{\phi\left(r_{0}\right) N(\varepsilon)+K \omega(\varepsilon)+\phi\left(r_{0}\right) M(\varepsilon)\right\} \\
&+K \phi\left(r_{0}\right)\left\{k \omega(x, \varepsilon)+\omega_{r_{0}}^{1}(f, \varepsilon)\right\} .
\end{aligned}
$$

Consequently, we derive the following inequality:

$$
\begin{aligned}
\omega(Q X, \varepsilon) \\
\leq \omega \\
\quad \times\left\{\phi\left(r_{0}\right) N(\varepsilon)+K \omega(\varepsilon)+\phi\left(r_{0}\right) M(\varepsilon)\right\} \\
+K \phi\left(r_{0}\right)\left\{k \omega(X, \varepsilon)+\omega_{r_{0}}^{1}(f, \varepsilon)\right\} .
\end{aligned}
$$

Now, taking into account the fact that $\omega(\varepsilon) \rightarrow 0, M(\varepsilon) \rightarrow$ 0 , and $N(\varepsilon) \rightarrow 0$ as $\varepsilon \rightarrow 0$ and keeping in mind that the function $f$ is uniformly continuous on the set $I \times\left[-r_{0}, r_{0}\right]$, we derive from (49) the following estimate:

$$
\omega_{0}(Q X) \leq k K \phi\left(r_{0}\right) \omega_{0}(X) .
$$


From the above estimate, assumption (viii), and Theorem 3 , we infer that there exists at least one fixed point $x$ of the operator $Q$ in the ball $B_{r_{0}}$. Obviously, the function $x=x(t)$ is a solution of (18). This completes the proof.

In order to illustrate the result contained in Theorem 8, we provide an example.

Example 9. Let us consider the following nonlinear integral equation of Erdélyi-Kober type:

$$
\begin{aligned}
x(t)= & t \exp t \\
& +\frac{1}{\Gamma(1 / 2)} \int_{0}^{t} \frac{(4 / 3) s^{7 / 3}\left(t+\sin s^{2}+\sqrt[3]{x^{2}(s)}\right)}{\left(t^{4 / 3}-s^{4 / 3}\right)^{1 / 2}} d s
\end{aligned}
$$

for $t \in I=[0,1]$. At first, let us observe that this equation can be written in the form (6). Indeed, we have

$$
\begin{aligned}
x(t)= & t \exp t \\
& +\frac{1}{\Gamma(1 / 2)} \int_{0}^{t} \frac{(4 / 3) s^{1 / 3} s^{2}\left(t+\sin s^{2}+\sqrt[3]{x^{2}(s)}\right)}{\left(t^{4 / 3}-s^{4 / 3}\right)^{1 / 2}} d s .
\end{aligned}
$$

Thus, (52) is a particular case of (6) if we put $a(t)=t \exp t$, $\alpha=1 / 2, m=4 / 3, p=2$, and

$$
v(t, s, x)=t+\sin s^{2}+x^{2 / 3} .
$$

Further, let us notice that (52) can be treated as a particular case of Volterra-Stieltjes integral equation (18) if we take into account the fact that the function $g=g(t, s)$ appearing in (18) has the form (26); that is,

$$
g(t, s)=t^{2 / 3}-\left(t^{4 / 3}-s^{4 / 3}\right)^{1 / 2} .
$$

It is easily seen that such a function $g(t, s)$ satisfies assumptions (iii)-(vi) of Theorem 8 . Moreover, we see that $f(t, x) \equiv$ 1 and $|v(t, s, x)| \leq 2+x^{2 / 3}$.

Thus, applying Theorem 8 , we can accept that $\phi(r)=2+$ $r^{2 / 3}$. We omit further, technical details (cf. [25]) but the final conclusion asserts that (52) has a solution in the space $C(I)$ belonging to the ball $B_{4}$.

\section{Further Results and Remarks}

The result contained in Theorem 8 does not cover some cases being important with regard to applications. Obviously, we can also formulate a more general theorem than that presented above and concerning the existence of solutions of (18) which are defined, continuous, and bounded on $\mathbb{R}_{+}$and are satisfying some other conditions (e.g., having a limit at infinity).

On the other hand, we can always adapt a suitable version of Theorem 8 in combination with the considered particular class of integral equations discussed above.
For example, if we consider the Volterra-Wiener-Hopf integral equation (5), then its generalized Volterra-Stieltjes counterpart has the form

$$
x(t)=a(t)+\int_{0}^{t} v(s, x(s)) d_{s} g(t, s),
$$

with the function $g(t, s)$ of the form (24). Then, we can formulate the following existence result concerning (55) [21] (cf. also [36]).

Theorem 10. Assume that the following hypotheses are satisfied.

(i) The function $a=a(t)$ is continuous and bounded on $\mathbb{R}_{+}$. Moreover, there exists the limit $\lim _{t \rightarrow \infty} a(t)$ (of course, this limit is finite).

(ii) $v: \mathbb{R}_{+} \times \mathbb{R} \rightarrow \mathbb{R}$ is continuous and there exists $a$ function $\psi: \mathbb{R}_{+} \rightarrow \mathbb{R}_{+}$being nondecreasing on $\mathbb{R}_{+}$, $\psi(0)=0$, and $\lim _{t \rightarrow 0} \psi(t)=0$ such that

$$
|v(s, x)-v(s, y)| \leq \psi(|x-y|),
$$

for all $s \in \mathbb{R}_{+}$and $x, y \in \mathbb{R}$.

(iii) The function $s \rightarrow v(s, 0)$ is bounded on $\mathbb{R}_{+}$.

(iv) $g(t, s)=g: \Delta \rightarrow \mathbb{R}$ is uniformly continuous on the triangle $\Delta=\{(t, s): 0 \leq s \leq t\}$.

(v) The function $s \rightarrow g(t, s)$ is of bounded variation on the interval $[0, t]$ for each fixed $t \in \mathbb{R}_{+}$.

(vi) For any $\varepsilon>0$, there is $\delta>0$ such that, for all $t_{1}, t_{2} \in$ $\mathbb{R}_{+}, t_{1}<t_{2}$, and $t_{2}-t_{1} \leq \delta$, the inequality

$$
\bigvee_{s=0}^{t_{1}}\left[g\left(t_{2}, s\right)-g\left(t_{1}, s\right)\right] \leq \varepsilon
$$

holds.

(vii) $g(t, 0)=0$ for all $t \geq 0$.

(viii) The function $t \rightarrow \bigvee_{s=0}^{t} g(t, s)$ is bounded on $\mathbb{R}_{+}$.

(ix) There exists a positive solution $r_{0}$ of the inequality

$$
\|a\|+\left(\psi(r)+V_{1}\right) K \leq r
$$

where $\|a\|=\sup \{|a(t)|: t \geq 0\}, V_{1}=\sup \{|v(s, 0)|:$ $s \geq 0\}$, and $K=\sup \left\{\bigvee_{s=0}^{t} g(t, s): t \geq 0\right\}$.

Then, (55) has at least one solution $x=x(t)$ which is defined, continuous, and bounded on $\mathbb{R}_{+}$and has a finite limit at infinity.

Further, let us mention that the crucial role in Theorem 8 is played by assumption (v) (the same assumption appears as assumption (vi) in Theorem 10). That assumption seems to be rather difficult to be verified in practice. But it turns out that, in considerations which cover all our particular classes of the above indicated integral equations, we can replace the mentioned assumption by less restrictive ones which are very convenient in verification.

For example, we formulate below the assumption of such a type which is connected with Theorem 10 (see [21]). 
(x) For arbitrary $t_{1}, t_{2} \in \mathbb{R}_{+}$such that $t_{1}<t_{2}$, the function $s \rightarrow g\left(t_{2}, s\right)-g\left(t_{1}, s\right)$ is nonincreasing on the interval $\left[0, t_{1}\right]$.

Then, we have the following lemma [21].

Lemma 11. Let assumptions (iv) and (vii) of Theorem 10 be satisfied. Moreover, we assume that the function $g=g(t, s)$ satisfies condition ( $x$ ). Then, $g$ satisfies assumption (vi) of Theorem 10.

It can be shown that Lemma 11 enables us to formulate convenient requirements concerning, for example, the function $k=k(u)$ appearing in (6), which guarantee that the Volterra-Wiener-Hopf counterpart of (55) satisfies assumptions imposed in Theorem 10. We omit details which can be found in [21].

\section{Remarks concerning Nonlinear Volterra-Stieltjes Integral Equations in Two Variables}

In this final section, we indicate the possibility of investigations concerning the nonlinear Volterra-Stieltjes integral equations with an unknown function of two or more variables (cf. [31]). For example, the Volterra-Stieltjes integral equation in two variables has the form

$$
\begin{aligned}
u(t, x)= & a(t, x)+f(t, x, u(t, x)) \\
& \times \int_{0}^{t} \int_{0}^{x} v(t, s, x, y, u(s, y)) d_{y} g_{2}(x, y) d_{s} g_{1}(t, s),
\end{aligned}
$$

for $(t, x) \in I^{2}$, where $I=[0,1]$. Obviously, the interval $[0,1]$ can be replaced by any closed and bounded interval $[a, b]$.

We will not formulate in detail assumptions concerning the functions involved in (59). Those assumptions are combinations and a refinement of assumptions imposed in Theorem 8 (cf. [31]).

It is worthwhile mentioning that the Volterra-Stieltjes integral equation in two variables (59) covers a lot of particular cases being a combination of nonlinear integral equations of the type (3)-(6). For example, we can consider the functional integral equation with functions involved depending on two variables which has the form

$$
\begin{aligned}
u(t, x)= & a(t, x) \\
& +\frac{f(t, x, u(t, x))}{\Gamma(\alpha) \Gamma(\beta)} \int_{0}^{t} \int_{0}^{x} \frac{v(t, s, x, y, u(s, y))}{(t-s)^{1-\alpha}(x-y)^{1-\beta}} d s d y,
\end{aligned}
$$

for $t, x \in I$ and for $\alpha, \beta$ being fixed numbers in the interval $(0,1)$. Obviously, $(60)$ is a particular case of $(59)$ if we put

$$
\begin{gathered}
g_{1}(t, s)=\frac{1}{\alpha}\left[t^{\alpha}-(t-s)^{\alpha}\right], \\
g_{2}(x, y)=\frac{1}{\beta}\left[x^{\beta}-(x-y)^{\beta}\right],
\end{gathered}
$$

for $(t, s) \in \Delta_{1}$ and $(x, y) \in \Delta_{2}$, where $\Delta_{1}=\{(t, s): 0 \leq s \leq$ $t \leq 1\}$ and $\Delta_{2}=\{(x, y): 0 \leq y \leq x \leq 1\}$.

On the other hand, we can also consider the functional integral equation with functions depending on two variables, which has other mixed forms composed of functions $g=$ $g(t, s)$ appearing in previously investigated integral equations (3) $-(6)$.

Thus, we can consider the nonlinear Volterra-Stieltjes integral equation with an unknown function depending on two variables and having the form

$u(t, x)=a(t, x)$

$$
+\frac{f(t, x, u(t, x))}{\Gamma(\alpha)} \int_{0}^{t} \int_{0}^{x} \frac{t v(t, s, x, y, u(s, y))}{(t+s)(x-y)^{1-\alpha}} d s d y
$$

for $t, x \in I$ and for $\alpha$ being a fixed number in the interval $(0,1)$.

Observe that (62) is a particular case of (59) if we put

$$
\begin{gathered}
g(t, s)= \begin{cases}t \ln \frac{t+s}{t} & \text { for } 0<s \leq t \\
0 & \text { for } t=0,\end{cases} \\
g_{2}(x, y)=\frac{1}{\alpha}\left[x^{\alpha}-(x-y)^{\alpha}\right],
\end{gathered}
$$

for $(x, y) \in \Delta_{2}$.

Hence, we see that (62) represents the mixed type of Chandrasekhar and fractional order integral equations.

Obviously, it is not difficult to construct other mixed types of nonlinear integral equations with unknown functions in two variables which are particular cases of (59). For example, we can construct nonlinear integral equation in two variables of mixed type of Erdélyi-Kober and fractional order, of Erdélyi-Kober and Wiener-Hopf type, and so on.

The details are rather involved and we will not present details (cf. [31]).

\section{Conflict of Interests}

The authors declare that there is no conflict of interests regarding the publication of this paper.

\section{References}

[1] S. Chandrasekhar, Radiative Transfer, Oxford University Press, London, UK, 1950.

[2] E. T. Copson, "On an integral equation arising in the theory of diffraction," The Quarterly Journal of Mathematics, vol. 17, pp. 19-34, 1946.

[3] C. Corduneanu, Integral Equations and Applications, Cambridge University Press, Cambridge, UK, 1991.

[4] M. Garg, A. Rao, and S. L. Kalla, "Fractional generalization of temperature field problem in oil strata," Matematichki Bilten, no. 30, pp. 71-84, 2006.

[5] R. Hilfer, Applications of Fractional Calculus in Physics, World Scientific, River Edge, NJ, USA, 2000. 
[6] E. Hopf, Mathematical Problems of Radiative Equilibria, Cambridge Tract 31, Cambridge University Press, Cambridge, UK, 1934.

[7] S. Hu, M. Khavanin, and W. Zhuang, "Integral equations arising in the kinetic theory of gases," Applicable Analysis, vol. 34, no. 3-4, pp. 261-266, 1989.

[8] A. A. Kilbas, H. M. Srivastava, and J. J. Trujillo, Theory and Applications of Fractional Differential Equations, vol. 204, Elsevier Science, Amsterdam, Netherlands, 2006.

[9] R. K. Saxena and S. L. Kalla, "On a fractional generalization of the free electron laser equation," Applied Mathematics and Computation, vol. 143, no. 1, pp. 89-97, 2003.

[10] H. M. Srivastava and R. K. Saxena, "Operators of fractional integration and their applications," Applied Mathematics and Computation, vol. 118, no. 1, pp. 1-52, 2001.

[11] V. E. Tarasov, Fractional Dynamics: Application of Fractional Calculus to Dynamics of Particles, Fields and Media, Nonlinear Physical Science, Springer, Heidelberg, Germany; Higher Education Press, Beijing, China, 2010.

[12] P. P. Zabrejko, A. I. Koshelev, M. A. Krasnosel'skii, S. G. Mikhlin, L. S. Rakovschik, and J. Stetsenko, Integral Equations, Nordhoff, Leyden, Mass, USA, 1975.

[13] I. K. Argyros, "On a class of quadratic integral equations with perturbation," Functiones et Approximatio Commentari Mathematici, vol. 20, pp. 51-63, 1992.

[14] J. Banaś and T. Zajaç, "A new approach to the theory of functional integral equations of fractional order," Journal of Mathematical Analysis and Applications, vol. 375, no. 2, pp. 375387, 2011.

[15] J. Caballero and M. A. a. Darwish, "Solvability of a fractional hybrid initial value problem with supremum by using measures of noncompactness in Banach algebras," Applied Mathematics and Computation, vol. 224, pp. 553-563, 2013.

[16] K. Diethelm, The Analysis of Fractional Differential Equations, vol. 2004 of Lecture Notes in Mathematics, Springer, Berlin, Germany, 2010.

[17] V. Lakshmikantham, S. Leela, and J. Vasundara Devi, Theory of Fractional Dynamic Systems, Cambridge Scientific Publishers, Cambridge, UK, 2009.

[18] K. S. Miller and B. Ross, An Introduction to the Fractional Calculus and Fractional Differential Equations, John Wiley \& Sons, New York, NY, USA, 1993.

[19] I. Podlubny, Fractional Differential Equations, vol. 198 of Mathematics in Science and Engineering, Academic Press, San Diego, Calif, USA, 1999.

[20] S. G. Samko, A. A. Kilbas, and O. I. Marichev, Fractional Integrals and Derivatives, Gordon and Breach Science, Amsterdam, The Netherlands, 1993.

[21] N. K. Ashirbayev, J. Banaś, and A. Dubiel, "Solvability of an integral equation of Volterra-Wiener-Hopf type," Abstract and Applied Analysis, vol. 2014, Article ID 982079, 9 pages, 2014.

[22] J. F. Carlson and A. E. Heins, "The reflection of an electromagnetic plane wave by an infinite set of plates. I," Quarterly of Applied Mathematics, vol. 4, pp. 313-329, 1947.

[23] E. T. Copson, "On an integral equation arising in the theory of diffraction," The Quarterly Journal of Mathematics, vol. 17, pp. 19-34, 1946.

[24] J. A. Alamo and J. Rodriguez, "Operational calculus for modified Erdélyi-Kober operators," Serdica. Bulgaricae Mathematicae Publicationes, vol. 20, no. 3-4, pp. 351-363, 1994.
[25] N. K. Ashirbayev, J. Banaś, and R. Bekmololayeva, “On solutions of a nonlinear Erdélyi-Kober integral equation," Abstract and Applied Analysis, vol. 2014, Article ID 184626, 7 pages, 2014.

[26] M. A. Darwish and K. Sadarangani, "On Erdélyi-Kober type quadratic integral equation with linear modification of the argument," Applied Mathematics and Computation, vol. 238, pp. 30-42, 2014.

[27] A. Erdélyi, "On fractional integration and its application to the theory of Hankel transforms," The Quarterly Journal of Mathematics, vol. 11, no. 1, pp. 293-303, 1940.

[28] A. Erdélyi and H. Kober, "Some remarks on Hankel transforms," The Quarterly Journal of Mathematics, vol. 11, pp. 212-221, 1940.

[29] H. H. Hashem and M. S. Zaki, "Carathéodory theorem for quadratic integral equations of Erdélyi-Kober type," Journal of Fractional Calculus and Applications, vol. 4, no. 1, pp. 56-72, 2013.

[30] J. Wang, C. Zhu, and M. Fečkan, "Solvability of fully nonlinear functional equations involving Erdélyi-Kober fractional integrals on the unbounded interval," Optimization, vol. 63, no. 8, pp. 1235-1248, 2014.

[31] M. A. Darwish and J. Banaś, "Existence and characterization of solutions of nonlinear Volterra-Stieltjes integral equations in two variables," Abstract and Applied Analysis, vol. 2014, Article ID 618434, 11 pages, 2014.

[32] J. Appell, J. Banaś, and N. Merentes, Bounded Variation and Around, vol. 17 of Series in Nonlinear Analysis and Applications, Walter de Gruyter, Berlin, Germany, 2014.

[33] J. Banaś and K. Goebel, Measures of noncompactness in Banach spaces, vol. 60 of Lecture Notes in Pure and Applied Mathematics, Marcel Dekker, New York, NY, USA, 1980.

[34] R. R. Akhmerov, M. I. Kamenskii, A. S. Potapov, A. E. Rodkina, and B. N. Sadovskii, Measures of Noncompactness and Condensing Operators, vol. 55 of Operator Theory: Advances and Applications, Birkhäuser, Basel, Switzerland, 1992.

[35] J. Appell and P. P. Zabrejko, Nonlinear Superposition Operators, vol. 95 of Cambridge Tracts in Mathematics, Cambridge University Press, Cambridge, UK, 1990.

[36] T. Zając, "Solvability of fractional integral equations on an unbounded interval through the theory of Volterra-Stieltjes integral equations," Zeitschrift für Analysis und ihre Anwendungen, vol. 33, no. 1, pp. 65-85, 2014. 


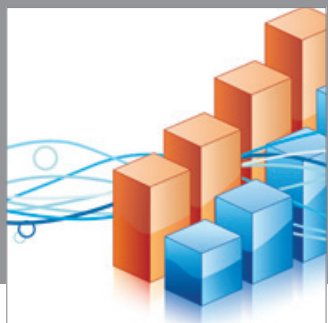

Advances in

Operations Research

mansans

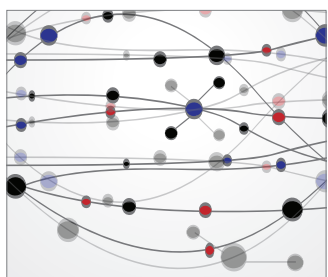

The Scientific World Journal
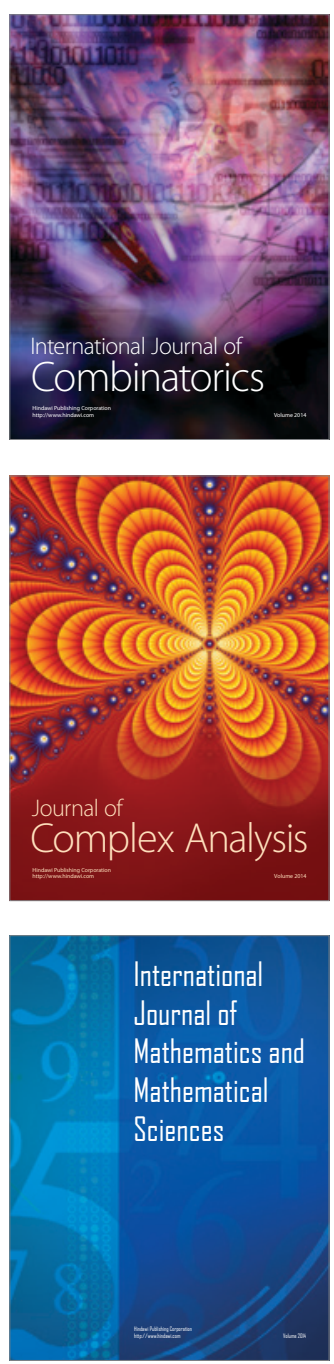
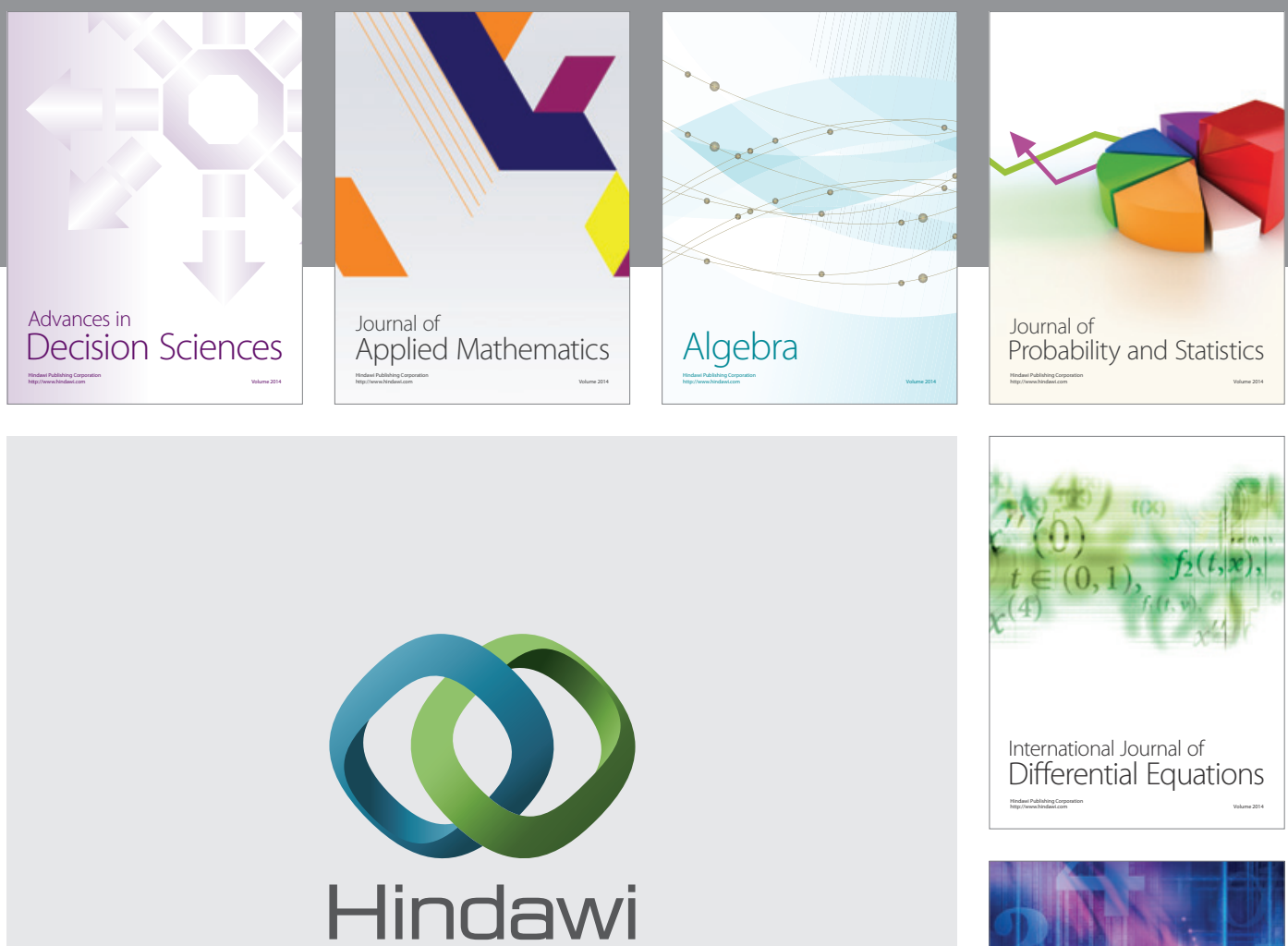

Submit your manuscripts at http://www.hindawi.com
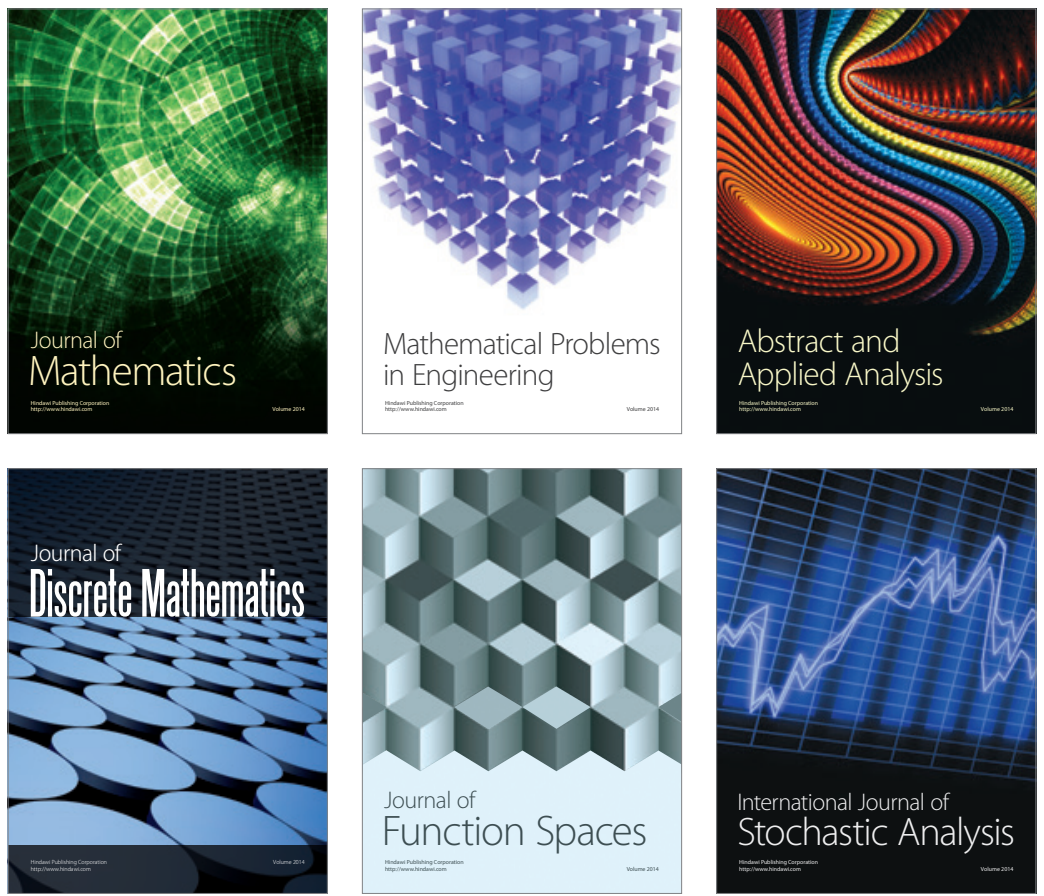

Journal of

Function Spaces

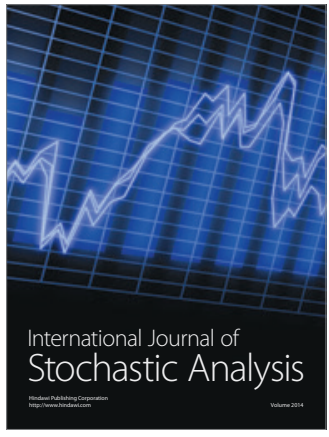

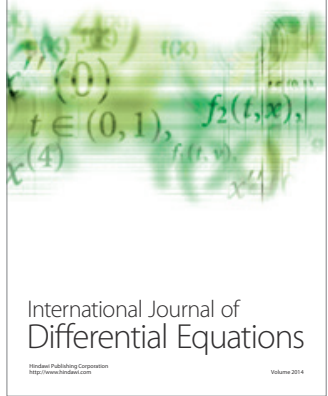
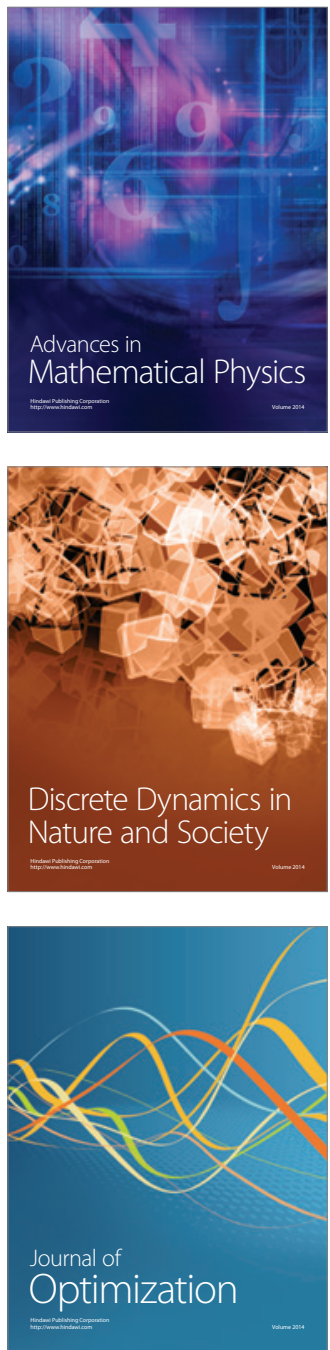